\title{
Vibration Analysis and Time Series Prediction for Wind Turbine Gearbox Prognostics
}

\author{
Sajid Hussain ${ }^{1}$ and Hossam A. Gabbar ${ }^{1,2}$ \\ ${ }^{1}$ Faculty of Engineering and Applied Science, University of Ontario Institute of Technology, 2000 Simcoe St. North, Oshawa, \\ Ontario, Canada L1H7K4 \\ sajid.hussain@uoit.ca \\ ${ }^{2}$ Faculty of Energy Systems and Nuclear Science, University of Ontario Institute of Technology, 2000 Simcoe St. North, \\ Oshawa, Ontario, Canada L1H7K4
}

hossam.gaber@uoit.ca

\begin{abstract}
Multiple premature failures of a gearbox in a wind turbine pose a high risk of increasing the operational and maintenance costs and decreasing the profit margins. Prognostics and health management (PHM) techniques are widely used to assess the current health condition of the gearbox and project it in future to predict premature failures. This paper proposes such techniques for predicting gearbox health condition index extracted from the vibration signals. The progression of the monitoring index is predicted using two different prediction techniques, adaptive neuro-fuzzy inference system (ANFIS) and nonlinear autoregressive model with exogenous inputs (NARX). The proposed prediction techniques are evaluated through sun-spot dataset and applied on vibration based health related monitoring index calculated through psychoacoustic phenomenon. A comparison is given for their prediction accuracy. The results are helpful in understanding the relationship of machine conditions, the corresponding indicating features, the level of damage/degradation, and their progression.
\end{abstract}

\section{INTRODUCTION}

There is a growing interest in renewable energy systems with increased concerns over climate change. Wind energy has an attractive share in renewable energy because it diversifies a resource portfolio and improves overall security of the power system. However, the engineering challenge for the wind industry is to design a reliable wind turbine to harness wind energy and turn it into electricity. Despite all technological advancements in wind turbine design and installation, there is a price to pay in maintaining

Sajid Hussain et al. This is an open-access article distributed under the terms of the Creative Commons Attribution 3.0 United States License, which permits unrestricted use, distribution, and reproduction in any medium, provided the original author and source are credited. the wind turbine in harsh operating environments and reduced accessibility. According to two large surveys of European wind turbines, conducted over a span of 13 years, gearbox failure is one of the highest risk events in wind turbines (C. C. James, 2011). Hence, there is a need for efficient condition monitoring system for wind turbine gearbox. Condition monitoring is a good tool to assess the damage early in time in order to plan the maintenance activities in a better way. Condition monitoring can be combined with opportunity maintenance to reduce the turbine's unexpected downtimes.

Typically, faults in wind turbine gearbox arise while in operation. Therefore, it is vital to detect, diagnose and analyze these faults as early as possible. The process should be non-destructive in nature to avoid wind turbine's disassembly. This study presents fault detection, features extraction, and prognostics for wind turbine gearbox based on vibration analysis. Vibration analysis is a non-destructive testing (NDT) technique widely used in the industry and in academia.

\subsection{Classification of Vibration Signals}

Vibration signals emanating from the rotating gearbox are analyzed to ascertain the current condition of the gearbox. Vibration signals can be classified into stationary and nonstationary, and based on this classification, the nature of their analysis methods differ. For stationary signals, vibration analysis methods are divided into two domains, namely time and frequency. Time-domain methods include statistical, model based, and signal processing based methods. Frequency-domain methods include spectrum and cepstrum based methods. For non-stationary signals, joint time-frequency vibration analysis methods such as short time Fourier transform (STFT) and wavelet analysis (WA) (Hui Li et al., 2011) are used for fault detection in 
gearboxes. Other methods of non-stationary analysis for gearboxes include Wigner-Ville distribution, Hilbert-Haung transform, and kurtogram analysis (Jerome Antoni, 2007).

\subsection{Features Extraction}

The features extraction system extracts characteristic signatures from raw vibration signals emanating from gearbox. The extracted features should reflect the changes in the gearbox's health conditions over time. As discussed earlier, the vibration based signal processing analysis is one of the most common non-destructive techniques. Also, with suitable vibration analysis, we can detect many different faults related with gearboxes. We use vibration based features extraction to extract the information that best represents the faulty conditions present in the monitored equipment. Different methods for vibration based features extraction in gearbox fault diagnosis framework have been proposed in research (Halima, E. B. et al, 2008, J. Rafiee et al, 2010). In time-domain, vibration based features such as kurtosis and spectral kurtosis are extensively used (F. Combet et al, 2009). Other studies including statisticalbased and transient-based features detection are performed in the past (Hiram Firpi and George Vachtsevanos, 2008; V. Indira et al, 2010). A comprehensive list of time-domain and frequency-domain features for fault detection and diagnosis of gearboxes is discussed in (Yaguo Lei et al, 2010).

\subsection{Prognostics}

Prognostic plays a very important role in an accurate and reliable decision making. Prognostics can be used effectively in utilization and maintenance of machinery systems. Prognostics uses different machines health related indices including temperature (Jamie Coble et al, 2010), oildebris analysis (Richard Dupuis, 2010), acoustics, and vibration (Eric Bechhoefer et al, 2010). Among these, vibration based prognostics is quite common. In (B. Samanta and C. Nataraj, 2008), researchers have used different health monitoring indices in gearboxes such as gear-wear, gear-chipped, gear-crack, gear-pitting, and shaft misalignment for projecting the gearbox health information in future. They have used neuro-fuzzy approaches for modeling and prediction of gearbox dynamics. A comprehensive review on prognostics is presented in (Andrew K.S. et al, 2006), where decision making process based on diagnostics and prognostics is discussed. Prognostic is performed by estimating the temporal evolution of the features over time (Wang W., 2007). In vibration based prognostics, vibration signals emanating from sensitive components inside the gearbox are recorded, health related features are extracted, and time series prediction techniques are applied to the features trends for prognostics (L. Gelman et al, 2012). Statistical, evolutionary and soft computing approaches are used to estimate the predictors and the use of neural networks and neuro-fuzy methods are very common.
This paper proposes a novel method to extract machine's health related vibration features based on psychoacoustic phenomenon along with neural networks and neuro-fuzzy approaches for prognostics. In this study, other than vibration features, we also use sunspot data for measuring the performance of the designed predictors. The rest of the paper is organized as follows. Section 2 is the methodology section where we propose the novel feature extraction technique based on psychoacoustics phenomenon followed by wavelet smoothing and prediction approaches. Section 3 simulates the proposed techniques on sunspot data and realworld vibration data emanating from a planetary gearbox inside a wind turbine. Finally, section 4 concludes the paper.

\section{THE MeThODOLOGY}

The process of vibration based features extraction and prognostic is shown in Figure 1. Below we discuss each module one by one.

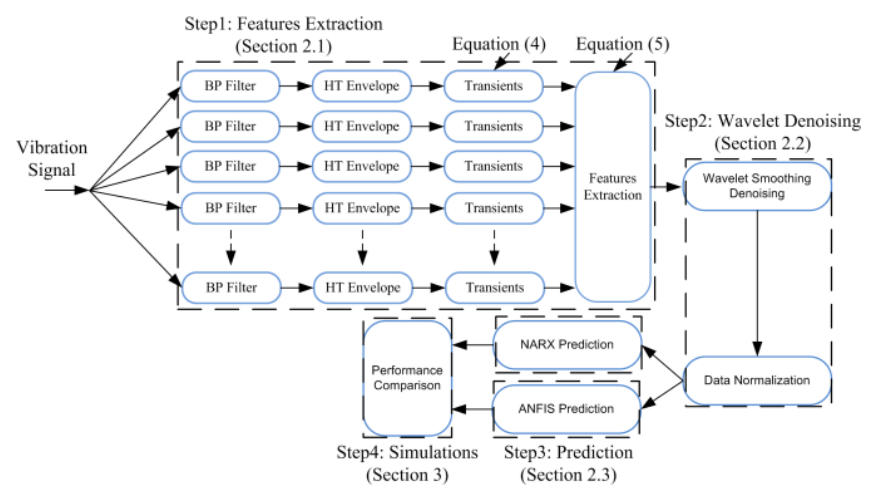

Figure 1. The process of prognostic

\subsection{Transient Based Features Extraction}

The vibration features extraction algorithm presented in this section works on the principle of transient analysis. Transients are very short and abrupt changes in sound waves due to non-linearity. Non-linearity could be mechanically introduced disturbances in electromechanical systems or unwanted clicks in transmission lines. The transient analysis algorithm calculates a real time estimate of transients caused by non-linearity as perceived by the human ear. The analyzer uses the knowledge about human ear's nature of filtering the signals as presented in (E. Zwicker, and $\mathrm{H}$. Fastl, 2009). The filtering operation ensures that the transients are detected in a way that matches the nature of the cochlea and thereby as perceived by the human ear. Transient analysis gives a much better correlation to the perceived quality of sound than traditional measurements based on frequency analysis.

The vibration features extraction algorithm uses principles of auditory models developed by the auditory physiologists (S. Seneff, 1988). According to psychoacoustic theory, pulses with short rise time or fall time contain a broad 
spectrum of frequencies. Therefore, it is possible to detect the instantaneous energy in frequency bands in the transient range of the ear. A common method for doing this is to use a filter bank containing a group of band-pass filters covering the frequency interval of interest. The purpose of the band pass filters is to detect the pulses in the frequency band where the pulses have most energy as perceived by the human ear. It will be the filter where the shape of the impulse response of the band-pass filter matches best the shape of the pulses but reverse in time. A gammatone filter bank is used in this study as shown in Figure 2. First designed by Patterson and Holdworth (R. D. Patterson et al, 1992), gammatone filter bank is an array of band-pass filters which simulates the response of the human ear's cochlea. At each point along the cochlea, a psychoacoustic measure of the width of the auditory filter is represented by an equivalent rectangular bandwidth (ERB).

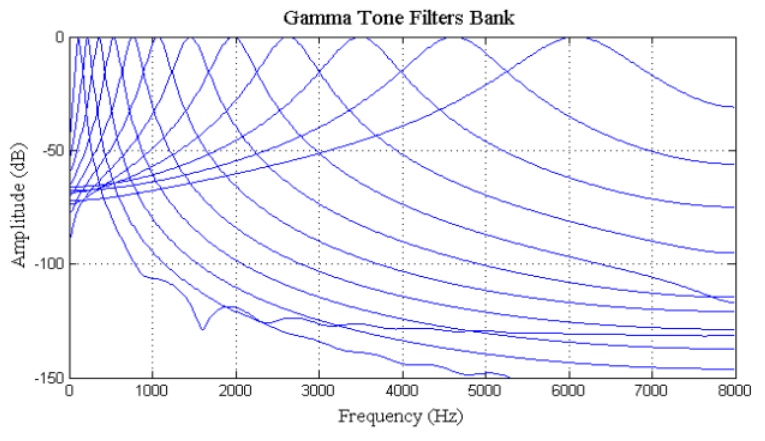

Figure 2. The gammatone filter bank

The ERB is a psychoacoustic measure of a filter's bandwidth in the filter bank. The bandwidth of a filter increases with an increase in its center frequency. The relationship between ERB and center frequency $F_{c} \mathrm{~Hz}$ is given by the following equation

$$
E R B=24.7+0.108 F_{c} .
$$

The impulse response of a band-pass filter is defined by the following relation (R. D. Patterson et al, 1992):

$$
h(t)=R t^{N-1} e^{-2 \pi m t} \cos \left(2 \pi F_{c} t+\phi\right) .
$$

Where $R$ is an arbitrary factor that is typically used to normalize the peak magnitude transfer to unity, $N$ is the filter order, $m$ is a parameter that determines the duration of the impulse response and thus the filter's bandwidth, $F_{c}$ is the filter's center frequency, and $\phi$ is the phase of the tone. Figure 3 shows an impulse response of a gammatone filter with $F_{c}=1000 \mathrm{~Hz}, m=125 \mathrm{~Hz}$ and $N=4$. To detect the energy in the channels the output signals from the band-pass filter bank are Hilbert transformed. Hilbert transform detects the envelopes of the band-pass filtered signals and extracts the instantaneous energy of the faulty pulses. Hilbert transform can be expressed as

$$
\hat{x}(t)=x(t) * \frac{1}{\pi t}=\frac{1}{\pi} \int_{-\infty}^{\infty} \frac{x(\tau)}{t-\tau} d \tau .
$$

Hilbert transform creates an artificially complex signal $u(t)$ from $x(t)$. The real part $x(t)$ of the $u(t)$ is the original signal and the imaginary part $\hat{x}(t)$ is the Hilbert transform of the real part. Thus, $u(t)$ is defined as $u(t)=x(t)+j \hat{x}(t)$. The magnitude and phase of $u(t)$ is computed as $A(t)=\sqrt{x(t)^{2}+\hat{x}(t)^{2}}$ and $\theta(t)=\arctan ^{\hat{x}(t)} / x(t)$. The magnitude $A(t)$ is the envelope of the signal and is always a positive function.

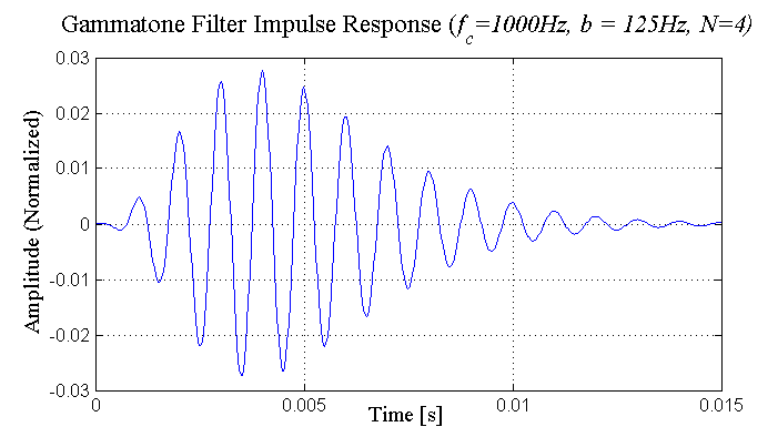

Figure 3. Gammatone filter impulse response

After the pulses are extracted through envelope detection or Hilbert transform, feature extraction block follows. In the feature extraction block, amplitudes and slopes of the pulses are calculated as per Eq. (4) and Figure 4.

$$
S_{r}=\frac{\text { High Reference Level-Low Reference Level }}{\text { Rise Time }}
$$

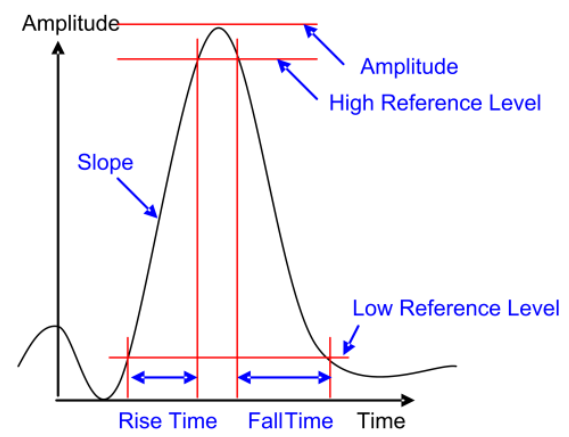

Figure 4. Calculation of amplitude and slope of a pulse

Amplitude (maximum magnitude) is a linear detector for pulses in the full frequency area. Slope is very sensitive for catching nonlinear sounds in the high frequency area. Both 
metrics can be summarized to a logarithmic index expressing the amount and size of the pulses. The index may be based on either the magnitudes or the slopes of the pulses. It is expected that pulses with a short rise time will be more annoying to the ear, than pulses with longer rise times. Thus, by considering the pulse envelope, it seems reasonable to focus the measure on the slope in the leading edge. Every transient detected is characterized by the $80 \%$ amplitude and the maximum slope in the leading edge. In order to obtain an equal number of detected transients in each frequency bands, the output from the transient analyzer is split into blocks of a pre-specified duration. The duration is found as a trade-off between preserving the complexity of the measurement and having an equal number of detected transients in each channel. In each block, only the transient with the maximum slope is gathered. The maximum slope is found by differentiating the signal and finding maximum amplitude of the differentiated signal. The logarithmic index is calculated as follows

$$
V b_{S}=10 . \log _{10}\left(\frac{\frac{1}{N_{B} N_{C}} \sum_{i, \text { channels }} \sum_{j=1}^{N_{B}}\left(S_{\text {max }}\right)_{i j}^{2}}{S_{\text {ref }}^{2}}\right) .
$$

Where $V b_{S}$ is vibration steepness index, $i$ is index for band pass filter and $N_{C}$ is total number of band pass filters used. Index of sub-blocks in a band pass filtered signal is $j$ and $N_{B}$ is the total number of sub-blocks. The maximum steepness in band pass filter $i$ and sub-block $j$ is $\left(S_{\max }\right)_{i j}$. The argument for squaring the maximum steepness in Eq. (5) is simply to put the large steepness values in favor. $S_{r e f}=1$ if the measured amplitude is acceleration. For amplitude index $V b_{A},\left(S_{\max }\right)_{i j}$ in Eq. (5) is replaced by $\left(A_{\max }\right)_{i j}$ where $A_{\max }$ is the maximum amplitude of the nondifferentiated envelope signal. The vibration features extracted are de-noised through wavelet de-noising techniques and normalized before prediction.

\subsection{Wavelet Denoising}

Features extracted from real signals emanating from complex dynamical systems pose a serious problem of noise. Therefore, it is important to de-noise the extracted features before modeling is performed (Uros Lotric and Andrej Dobnikar, 2005). De-noising the signal is one of the most effective applications of wavelets in signal processing. The wavelet transform-based de-noising methods can produce much higher de-noising quality than conventional methods. Furthermore, the wavelet transform-based methods retain the details of a signal after de-noising (Edmundo G. de Souza e Silva et al, 2010). Wavelets are limited duration, undulatory mathematical functions. The time integral of wavelet functions equals to zero. Figure 5 plots some common wavelets. Similar to Fourier transform, where we use sines and cosines as basis functions, wavelet transform uses wavelets as basis functions. Wavelets are used in many different fields including compression, signal processing, and de-noising (Graps, A, 1995). In Fourier analysis, we approximate a function $f(x)$ by sines and cosines functions with different frequencies and amplitudes. Thus, the approximation equation becomes

$$
\hat{f}(x)=a_{o}+\sum_{k=1}^{\infty}\left(a_{i} \sin (k x)+b_{i} \cos (k x)\right) .
$$

Where, $a_{o}, a_{i}$, and $b_{i}$ are calculated from Fourier transform as

$$
F(\omega)=\int_{-\infty}^{\infty} f(t) e^{-j \omega t} d t
$$

Wavelet analysis is performed in the similar way as Fourier transform but with scaled and translated versions of mother wavelet $\psi(x)$ as basis functions. Mother wavelet can be any one from Figure 5 and the scaling and translation is defined as child wavelets and can be calculated as

$$
\psi_{j, k}(x)=\kappa \psi\left(2^{j} x-k\right) .
$$

Where $\kappa$ is a constant, $k$ is wavelet translation and $2^{j}$ is scale translation. We can estimate $f(x)$ from the following equation in wavelet analysis

$$
\hat{f}(x)=\sum_{\forall j, \forall k} c_{j, k} \psi_{j, k}(x) .
$$

Where, $c_{j, k}$ are the wavelet coefficients and are obtained through the wavelet transform as

$$
c_{j, k}=\int_{-\infty}^{\infty} f(x) \psi_{j, k}(x) d x .
$$

Each coefficient $c_{j, k}$ obtained in Eq. (10) is a contribution of the wavelet $\psi_{j, k}(x)$ in the whole approximation for the original signal. If the value of this coefficient $c_{j, k}$ is very small and its contribution to the approximation is considered negligible, we can omit the corresponding child wavelet $\psi_{j, k}(x)$ from the approximation. This procedure is called 
thresholding and it forms the basis for the wavelet denoising. Wavelet analysis basis functions are finite and limited to one size and this makes wavelet analysis useful technique for detecting local features like discontinuities and spikes in a signal. On the other hand, Fourier analysis basis functions are infinite in nature and an approximation of a specific part of the signal affects the entire signal. Wavelet analysis is joint time-frequency analysis technique as contrast to the Fourier transform that is purely frequency analysis. This feature makes wavelet analysis to detect when in time a particular event took place. Wavelet smoothing or wavelet trend analysis is used to remove high frequency components from the extracted features which can be assumed as noise.
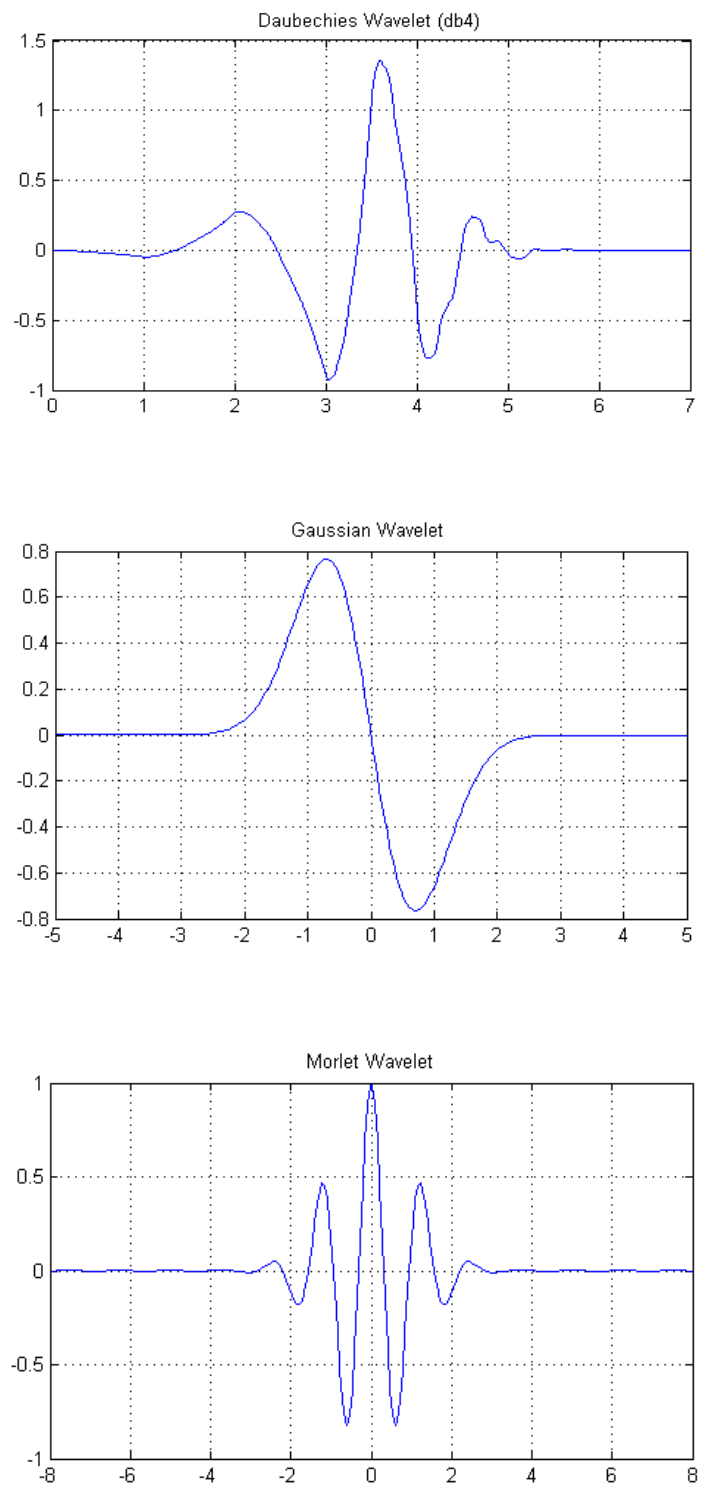

Figure 5. Commonly used wavelets (a) Daubechies (b) Gaussian (c) Morlet

\subsection{Time Series Prediction}

The prediction of time series $x(t)$ at $r$ time steps ahead, $x_{t+r}$, is obtained based on its values at present and past time

steps $\quad\left[x_{t-m r}, x_{t-(m-1) r}, x_{t-(m-2) r}, \ldots, x_{t-2 r}, x_{t-r}, x_{t}\right] \quad$ as $x_{t+r}=\chi\left(x_{t-m r}, x_{t-(m-1) r}, x_{t-(m-2) r}, \ldots, x_{t-2 r}, x_{t-r}, x_{t}\right)$. Where $\chi$ is a predictor functions and can be approximated through various conventional, statistical and artificially intelligent techniques like Bayesian, support vector regression, adaptive neuro-fuzzy inference system (ANFIS) and neural networks (NN). This paper uses a dynamic neural network called the nonlinear autoregressive model with exogenous inputs (NARX) and ANFIS techniques to approximate the predictor function $\chi$.

\subsubsection{The NARX}

The NARX is a dynamic neural network, used for modeling nonlinear dynamical systems. The NARX can be represented mathematically as

$$
y(n+1)=f\left[\begin{array}{l}
y(n), \ldots, y\left(n-d_{y}+1\right) \\
u(n), u(n-1), \ldots, u\left(n-d_{u}+1\right)
\end{array}\right] .
$$

Where, $\mathbf{u}(n)$ and $\mathbf{y}(n)$ are the input and output of the system at time step $n$, while $d_{u} \geq 1$ and $d_{y} \geq 1, d_{u} \leq d_{y}$, are the input-memory and output-memory orders. Equation (11) can also be written in compact form as $y(n+1)=f[\mathbf{y}(n) ; \mathbf{u}(n)]$, where $\mathbf{u}(n)$ and $\mathbf{y}(n)$ are the input and output regressor vectors respectively. The nonlinear mapping function $f($.$) is approximated through a$ multi-layer perception (MLP) algorithm trained with plain back propagation algorithm. This research deals with nonlinear univariate time series prediction and for this we set $d_{y}=0$. This reduces the NARX network to time delay neural network (TDNN) architecture and Eq. (11) reduces to (T. Lin et al, 1997)

$$
y(n+1)=f\left[u(n), u(n-1), \ldots, u\left(n-d_{u}+1\right)\right] .
$$

Figure 6 shows the way the NARX is trained and tested. During the training phase, the feedback loops (dotted lines in Figure 6) are not used. During the testing or prediction phase, if multistep-ahead predictions are required, the output values are fed back to both the input regressor $\mathbf{u}(n)$ and the output regressor $\mathbf{y}(n)$ at the same time. Thus, the resulting predictive model contains two feedback loops, one for the input regressor and another for the output regressor. 


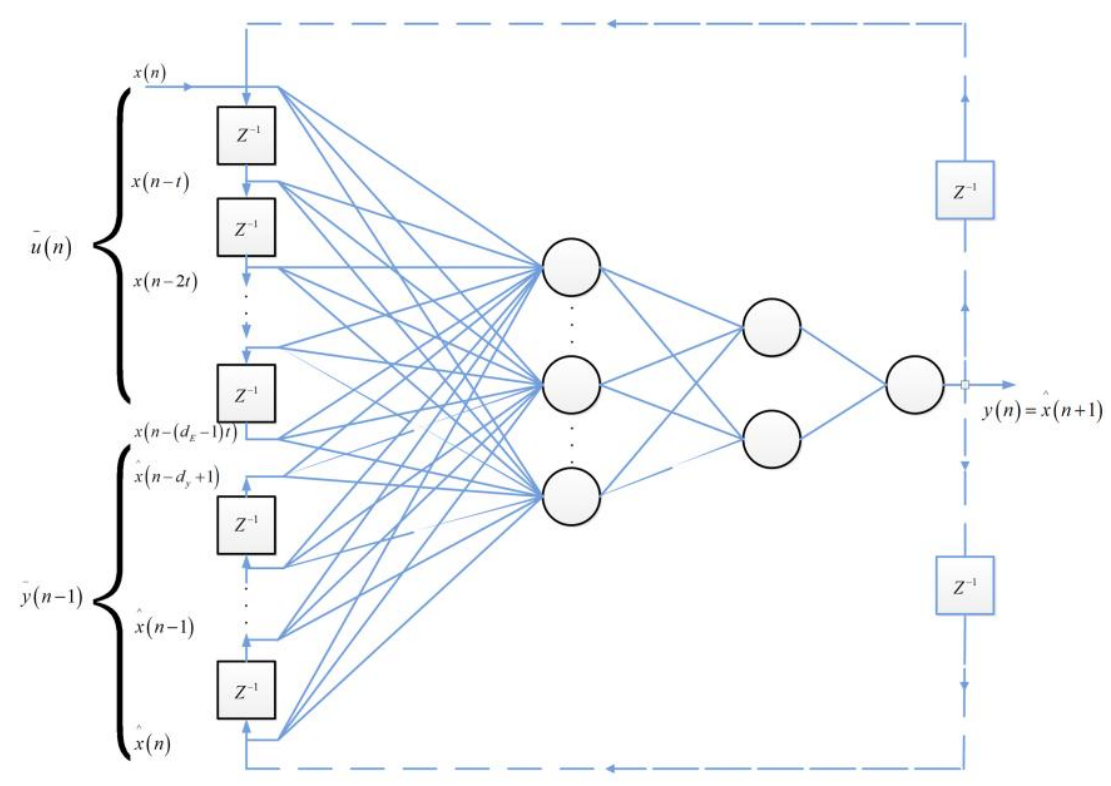

Figure 6. NARX training and testing

(Feedback loops are required only during testing)

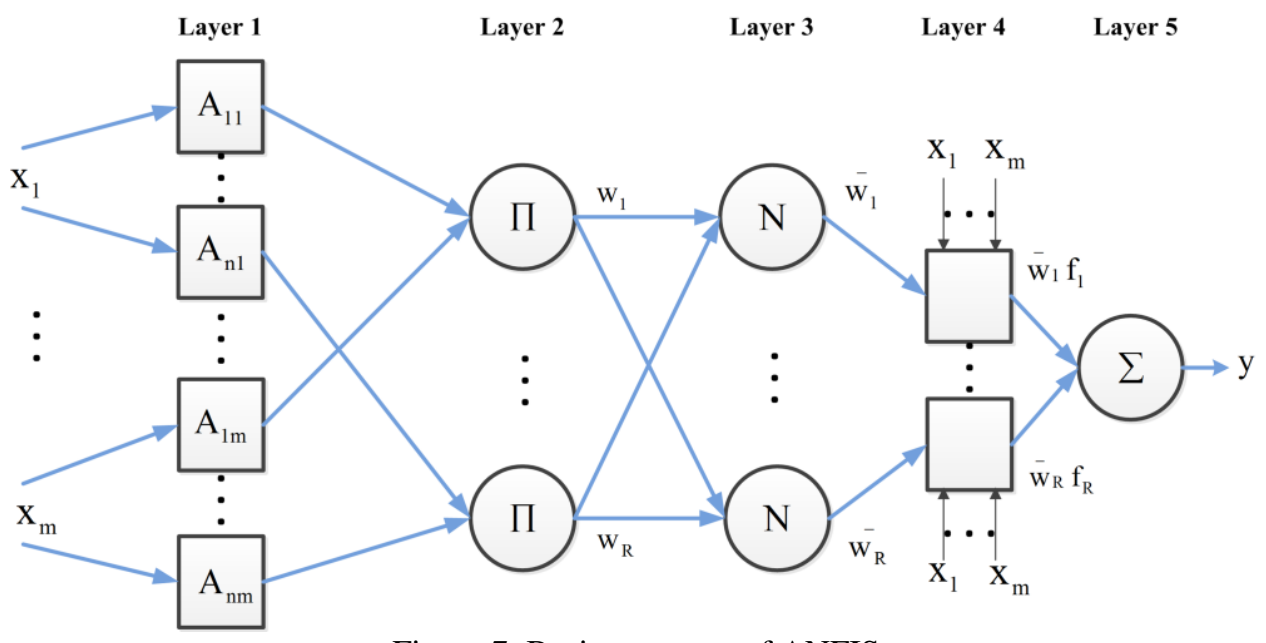

Figure 7. Basic structure of ANFIS

\subsubsection{The adaptive neuro-fuzzy inference system}

The basic structure of the ANFIS is shown in Figure 7. The ANFIS has $m$ inputs $\left(x_{1}, x_{2} \ldots, x_{m}\right)$, each with $n$ membership functions (MFs), $R$ rules and one output $y$. When the ANFIS predicts a time series, the inputs are $\left(x_{t-m r}, x_{t-(m-1) r}, x_{t-(m-2) r}, \ldots, x_{t-2 r}, x_{t-r}, x_{t}\right)$ and the output of the ANFIS is $y=x_{t+r}$. In the above mentioned case, the ANFIS predicts the time series $r$ time steps ahead based on current and the previous $m$ values. We use a Sugeno-fuzzy type inference system with five layers and $m=4$ inputs. Number of nodes $N$ in layer 1 is the product of number of inputs $m$ and the input MFs $n$ for each input, i.e., $N=m . n$ . Number of nodes in layers 2-4 is equal to the number of rules $R$ in the fuzzy rule base. Layer 1 is a fuzzufication layer and it transforms the crisp inputs $x_{i}$ to linguistic labels $A_{i j}$. The examples of the linguistic labels are small, medium, large etc., and the transformation occurs with some degree of the MFs as $O_{i j}^{1}=\mu_{i j}(x)$. Where, $i=1, \ldots, m$, $j=1, \ldots, n$ and $\mu_{i j}$ represents the $j$ th membership function for the input $x_{i}$. Different types of MFs are used like triangular, trapeziodal, Gaussian etc. Layer 2 of the ANFIS is a product layer, where for each node $k$, the output represents weighting factor or firing strength of the rule $R$ 
associated with $k$. The output $w_{k}$ of this layer is $O_{k}^{2}=\prod \mu_{i k}\left(x_{i}\right)$, and it is the product of all its inputs scaled according to the MFs $\mu_{i k}$.Where $i=1, \ldots, m$ and $k=1, \ldots, R$. Layer 3 is a normalization layer and the output of each node $k$ in this layer represents the normalized weighting factor $\bar{w}_{k}$ of the $k$ th rule as $O_{k}^{3}=\frac{w_{k}}{\sum_{k} w_{k}}$. Where $k=1, \ldots, R$. Layer 4 is a de-fuzzification layer and the output of each node in this layer is a weighted output of the first order Sugeno-type fuzzy if-then rule as $O_{k}^{4}=\overline{w_{k}} f_{k}$ . Where $f_{k}=\sum_{j} p_{k j} x_{j}+r_{k}, j=1, \ldots, n, k=1, \ldots, R, f_{k}$ is the output of the $k$ th rule, and the parameters $p_{k j}$ and $r_{k}$ are called consequent parameters. Layer 5 is the final output layer and it contains only one node inside. The output of the layer 5 is an overall output $y$ of the network as $O^{5}=\sum_{k} \bar{w}_{k} f_{k}$. It is also a sum of all the weighted outputs of the rules. We need a training dataset of desired input/output pairs $\left(x_{1}, x_{2}, \ldots, x_{m}, y\right)$ to train the ANFIS or model the target system. In training phase, the ANFIS adaptively maps the input features space $\left(x_{1}, x_{2}, \ldots, x_{m}\right)$ to the corresponding output $y$. The mapping in the ANFIS system is done through the membership functions (MFs), the rule base and the related parameters that emulate the training dataset. The training phase of the ANFIS uses hybrid learning method. It uses the gradient descent approach for fine tuning the parameters that define the MFs and applies the least squares method to identify the consequent parameters that define the coefficient of each output equation in Sugeno-type fuzzy rule base. The training process continues till the desired stopping criteria is reached, i.e., number of epochs or error tolerance.

\section{Simulations AND DiscuSSIONS}

In this section, the prediction accuracy of both the time series predictors, NARX and ANFIS is compared using standard dataset of sunspot activity for years 1749-2012 (RWC Belgium World Data Center, 2012). The sunspot activity data has non-linear, non-Gaussian and nonstationary characteristics and is suitable to test the performance of the predictors. The entire dataset of sunspot activity was normalized between $[0-1]$ and used for training and testing both NARX and ANFIS predictors. To get a reliable prognosis, the data need to be less sensitive to noise. This requirement can be reached using selected signal processing techniques such as wavelet smoothing or denoising discussed in section 2.2. For wavelet denoising, we use Daubechies wavelet $(\mathrm{db} 4)$ with nine levels. The threshold technique is set to soft threshold, and the threshold rule used is universal. The universal threshold rule is defined as $\sqrt{2 \times \log (L)}$. Where $L$ is the signal length. We also set the rescaling method as single level where the algorithm considers the noise as white and estimates the standard deviation of the noise from the wavelet coefficient at the first level. We use the normalized Akaike Information Criteria (AIC) for assessing the prediction performance. The AIC can be formulated as (Akaike H. 1974).

$$
A I C=\ln \sigma^{2}+\frac{2 u}{P} .
$$

Where $\sigma^{2}$ is the variance of the prediction error, $u$ is the number of model parameters to be updated and $P$ is the total number of data points in the predicted dataset. A smaller value of AIC indicates better prediction performance. The sunspot activity data contains 3166 points. For both types of predictors, $70 \%$ data is used for training, $15 \%$ for testing, $15 \%$ for validation, and 500 training epochs. Computation was carried out in MATLAB ${ }^{\circledR}$ environment on a PC with Intel Core 17 with 8GB of RAM. One step ahead prediction is performed and in one step ahead prediction, the target $y(n+1)$ is calculated from previous four values, $x(n), x(n-1), x(n-2)$, and $x(n-3)$

.The input memory we use is $d_{u}=4$. Table 1 shows the mean absolute error (MAE) and AIC for NARX and ANFIS for sunspot data. The number of membership functions for ANFIS is 3 with 16 fuzzy rules and 104 parameters. The AIC achieved is -4.457 . The neuro-fuzzy system proposed in (Wang WQ 2004) with the same datasets produced AIC of 1.527. The ANFIS method gave better prediction performance as compared to (Wang WQ 2004) for the same datasets. The sunspot training and test datasets were also used for assessing the prediction performance of NARX. For NARX, MAE of testing is 0.0034 .

Figure 8(a) shows the sunspot activity data along with wavelet smoothing. NARX and ANFIS prediction results are shown in Figure 8(b) and 8(c) along with error box-plots in Figure 8(d), respectively. In Figure 8(d), the medians of the box-plots are centered at zero for both NARX and ANFIS predictors and the $\pm 2.698 \sigma$ lines are at \pm 0.01 showing about $99.3 \%$ of the error observations within the range of \pm 0.01 . Where $\sigma$ is the error's standard deviation. There are some outliers for both NARX and ANFIS cases and NARX depicts better performance as compared to ANFIS in this case. In the case of NARX, the MAE value is approximately same as ANFIS (sunspot data-set) in Table 1.

In the framework of machine condition monitoring, it is very important to know well in advance the expected behavior of a machine system for its proper operation and 
maintenance scheduling without any major interruptions. Several approaches are adopted to monitor the system behavior and vibration-based approaches are quite popular for condition monitoring.
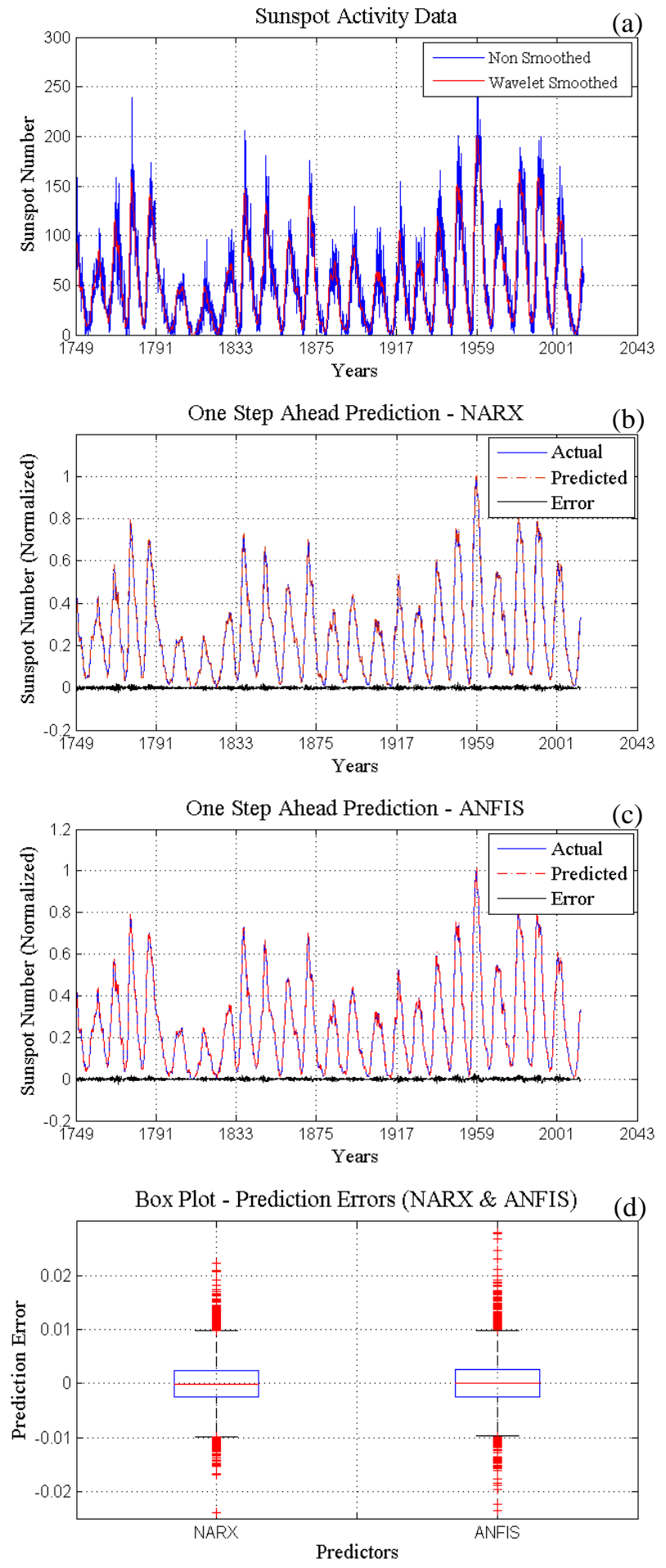

Figure 8. Sunspot data, wavelet smoothing and prediction
The desirable monitoring index should be highly sensitive to the fault-related vibration with low sensitivity to noise. The selection of monitoring index involves different steps of signal processing and feature extraction. These also greatly depend on the type of machine faults. The vibration index we use is proposed in section 2.1. The experimental vibration data emanate from a planetary gearbox inside a wind turbine. The data are provided by the National Renewable Energy Laboratory (NREL), through a consortium called the Gearbox Reliability Collaborative (GRC) (H. Link et. al. 2011). The gearbox under test is one of two units taken from the field and redesigned, rebuilt and instrumented with over 125 sensors. The gearbox first finished its run-in in the NREL dynamometer test facility (DTF) and later was sent to a wind plant close to NREL for field test, where two oil losses occurred.

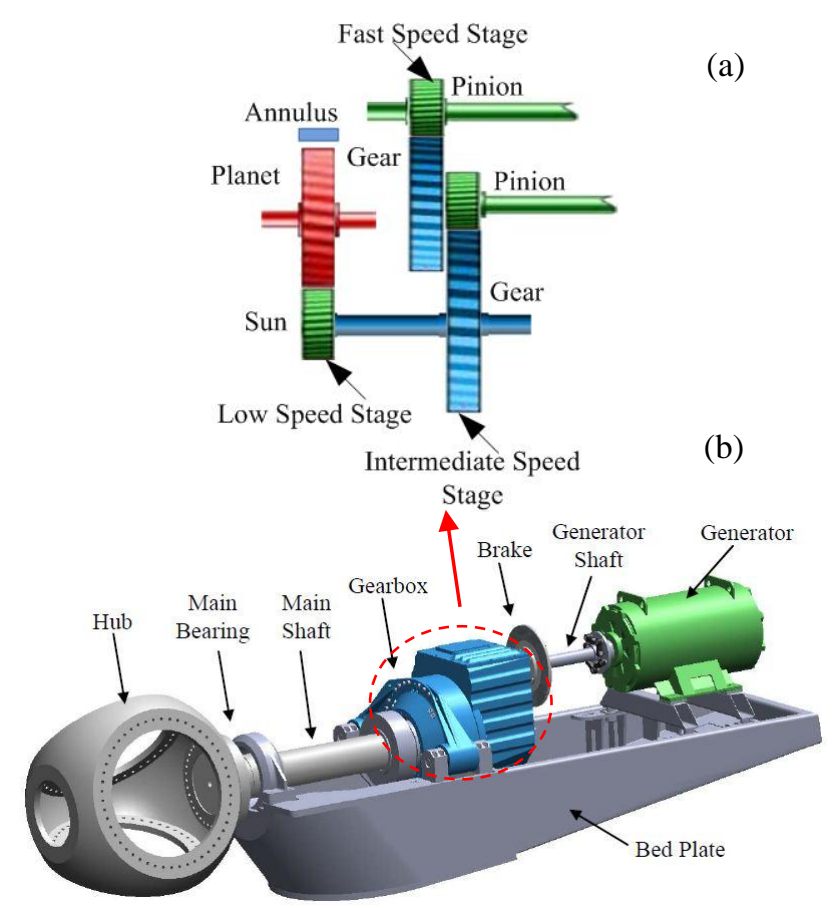

Figure 9. (a) Planetary gearbox (b) GRC Drive train Configuration

(Courtesy of National Renewable Energy Laboratory)

The test turbine in the field is a stall-controlled, threebladed, upwind turbine with a rated power of $750 \mathrm{~kW}$. The turbine generator operates at 1200 RPM and 1800 RPM nominal on two different sets of windings depending on the power. The planetary gearbox has an overall ratio of $1: 81.491$. It is composed of one low speed (LS) planetary stage and two parallel stages as shown in Figure 9. This study uses data from test case CM_2a with main shaft speed of 14.72 RPM and high speed shaft (HSS) speed of 1200 RPM. The data are collected at a sampling frequency of $F_{s}=40 \mathrm{KHz}$. Figure 10 (a) shows an example of a raw 
vibration signal, 1sec (40000 samples) in length, collected from the gearbox inside the windmill.
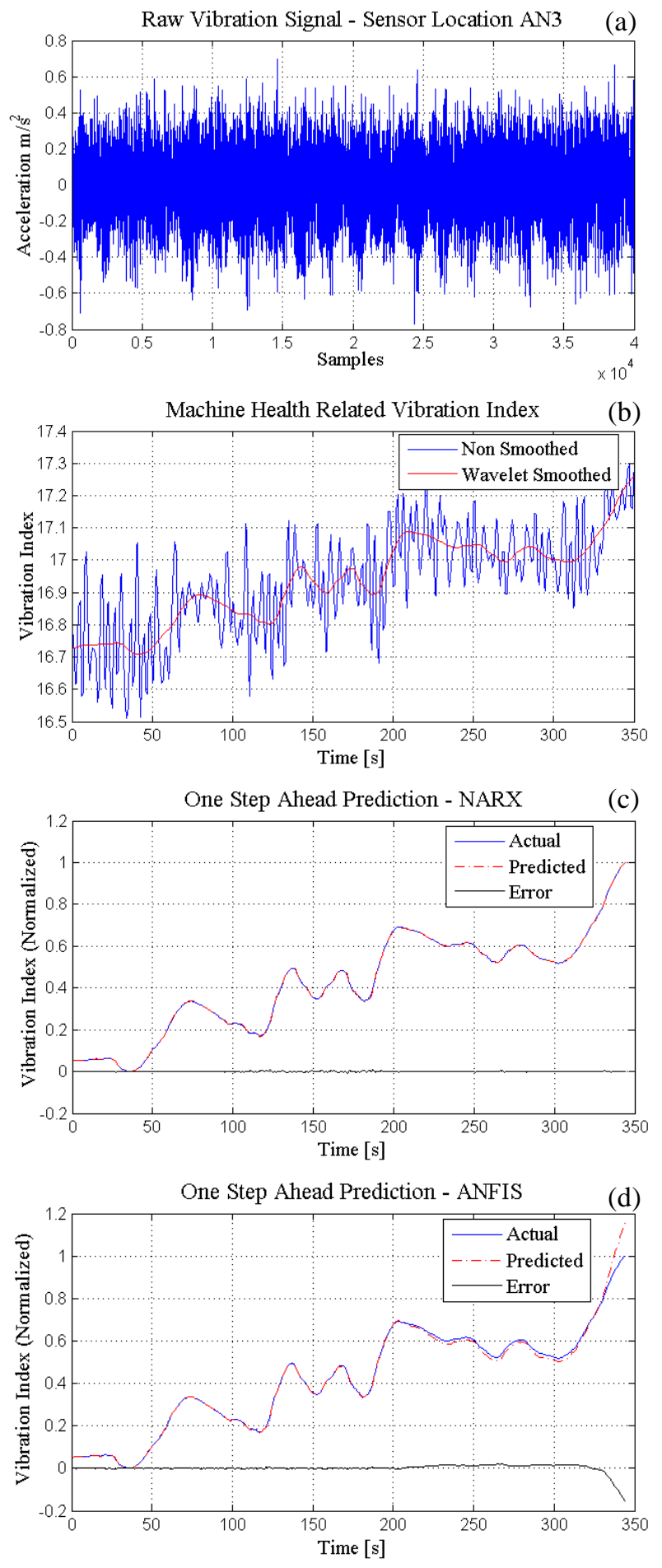

Figure 10. (a) Raw vibration signal (one sample) (b) Vibration index trend (350 samples) (c) one-step prediction NARX (d) one-step prediction ANFIS

A total of 350 such vibration signals are analyzed in this section. Vibration indices are calculated as proposed in section 2.1. For both types of predictors, $70 \%$ data is used for training, $15 \%$ for testing, $15 \%$ for validation, and 500 training epochs. Computation was carried out in MATLAB ${ }^{\circledR}$ environment on a PC with Intel Core i7 with $8 \mathrm{~GB}$ of RAM. Figure 10(a) shows an example of a raw vibration signal, $1 \mathrm{sec}$ (40000 samples) in length, collected from the gearbox inside the windmill. A total of 350 such vibration signals are analyzed in this section. Vibration indices are calculated as proposed in section 2.1. Figure 10(b) shows the vibration index trend with wavelet smoothing. The vibration index is gradually increasing with time. It is because of the oil loss occurred in the field test. The oil loss caused the gearbox to run dry and consequently, a gradual increase in the overall vibration levels and vibration index is observed. Figure 10(c) shows one step prediction for NARX and Figure 10(d) shows one step prediction for ANFIS. In one step ahead prediction, the target $y(n+1)$ is calculated from previous four values, $x(n), x(n-1), x(n-2)$, and $x(n-3)$.The input memory we use is $d_{u}=4$.
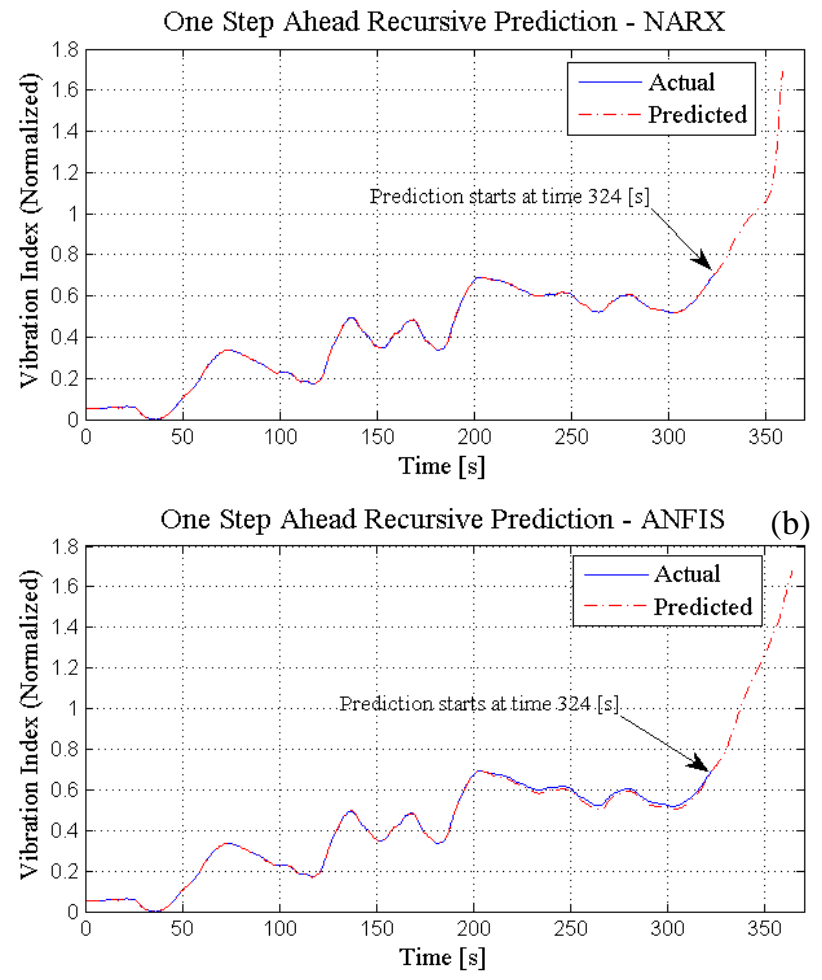

Figure 11. One-step ahead recursive prediction (a) NARX (b) ANFIS

NARX seems to exhibit more promising results as compared to ANFIS in this case. Also, the MAE error value for NARX is less as shown in Table 1 (vibration index dataset). We can also use one step recursive prediction for NARX and ANFIS to predict as many future values as we want as shown in Figures 11(a) and 11(b). For multi-step recursive prediction, we have to loop the output values back as discussed in section 2.3.1 in the NARX case (feedback 
dotted lines in Figure 6). Similar strategy is adopted in the ANFIS case.

\section{CONCLuSION}

Two different techniques are used for predicting the wind turbine gearbox health related vibration based index trend. The prediction performance of the predictors, NARX and ANFIS, is illustrated on two data-sets, sunspot activity and vibration index. Both the NARX and ANFIS predictors perform quit well in this study. Results show the effectiveness of the predictors in estimating the variations of the monitoring indices. In this work, one-step-ahead and recursive multi-step ahead prediction is considered for both the NARX and ANFIS cases. Although, the $k$ step ahead prediction is not performed in this research, it can be done easily by predicting the target $y(n+k)$ with the previous values $x(n), x(n-k), x(n-2 k), x(n-3 k)$ and so on. The potential application of these techniques for the development of on-line prognostic and estimation of remaining useful life (RUL) for machine condition is under consideration for further work.

\begin{tabular}{|c|c|c|c|c|}
\hline Data Set & Model & MAE & MSE & AIC \\
\hline \multirow{3}{*}{ Sunspot } & NARX & 0.0034 & $2.144 \times 10^{-5}$ & -4.943 \\
\cline { 2 - 5 } & ANFIS & 0.0035 & $2.421 \times 10^{-5}$ & -4.457 \\
\hline \multirow{2}{*}{$\begin{array}{c}\text { Vibration } \\
\text { Index }\end{array}$} & NARX & 0.0013 & $3.626 \times 10^{-6}$ & -3.467 \\
\cline { 2 - 5 } & ANFIS & 0.0086 & $4.079 \times 10^{-4}$ & -3.134 \\
\hline
\end{tabular}

Table 1. MAE, MSE ans AIC - NARX and ANFIS

\section{ACKNOWLEDGEMENTS}

Authors and thankful to the National Renewable Energy Laboratory (NREL) for providing the wind turbine's gearbox vibration data through a consortium called the Gearbox Reliability Collaborative (GRC).

\section{REFERENCES}

Akaike H. A new look at the statistical model identification (1974). IEEE Trans Automat Control. vol.17, pp.71623.

Andrew K.S. Jardine, Daming Lin, and Dragan Banjevic (2006). A review on machinery diagnostics and prognostics implementing condition-based maintenance. Mechanical Systems and Signal Processing, vol. 20, no.7, pp. 1483-1510.

B. Samanta and C. Nataraj (2008). Prognostics of machine condition using soft computing. Robotics and Computer-Integrated Manufacturing, vol. 24, no. 6, pp. 816-823.

C. C. James., (2011). Condition monitoring techniques for wind turbines. Doctoral dissertation, Durham University, UK. http://etheses.dur.ac.uk/652/.
E. Zwicker, and H. Fastl (2009). Psychoacoustics - Facts and Models. Springer $2^{\text {nd }}$ edition.

Edmundo G. de Souza e Silva, Luiz F.L. Legey and Edmundo A. de Souza e Silva (2010). Forecasting oil price trends using wavelets and hidden markov models. Energy Economics, vol. 32, pp. 1507-1519.

Eric Bechhoefer, Steve Clark, and David He (2010). A state-space model for vibration based prognostics. Annual Conference of the Prognostics and Health Management Society, October 10-16, Portland, Oregon USA.

F. Combet, L. Gelman (2009). Optimal filtering of gear signals for early damage detection based on the spectral kurtosis. Mechanical Systems and Signal Processing, vol. 23, no. 3, pp. 652-668.

Graps, A. (1995). An introduction to wavelets. IEEE Computational Science and Engineering, vol. 2, no. 2, PP. 50-61.

H. Link, W. LaCava, J. van Dam, B. McNiff, S. Sheng, R. Wallen, M. McDade, S. Lambert, S. Butterfield, and F. Oyague (2011). Gearbox reliability collaborative project report: Findings from Phase 1 and Phase 2 Testing. NREL/TP-5000-51885.

Hui Li, YupingZhang, and Haiqi Zheng (2011). Application of Hermitian wavelet to crack fault detection in gearbox. Mechanical Systems and Signal Processing, vol. 25, pp. 1353-1363.

Halima, E. B., Shoukat Choudhury, M. A. A., Shah, S. L., and Zuo, M. J. (2008). Time domain averaging across all scales: a novel method for detection of gearbox faults. Mechanical Systems and Signal Processing, vol. 22, no. 2, pp. 261-278.

Hiram Firpi and George Vachtsevanos (2008). Genetically programmed-based artificial feature-extraction applied to fault detection. Engineering Applications of Artificial Intelligence, vol. 21, no. 4, pp. 558-568.

J. Rafiee, M.A. Rafiee, P.W. Tse (2010). Application of mother wavelet functions for automatic gear and bearing fault diagnosis. Expert Systems with Applications, vol. 37, no. 6, pp. 4568-4579.

Jerome Antoni (2007). Fast computation of the kurtogram for the detection of transient faults. Journal of Mechanical systems and Signal Processing, vo. 21, no. 1, pp. 108-124.

L. Gelman, I. Petrunin, I. K. Jennions, and M. Walters (2012). Diagnostics of local tooth damage in gears by the wavelet technology. International Journal of Prognostic and Health Management, vol. 3, no. 2.

R. D. Patterson, K. Robinson, J. Holdsworth, D. McKeown, C.Zhang, and M. H. Allerhand (1992). Complex sounds and auditory images. Auditory physiology and perception, Proc. 9th International Symposium on Hearing, Pergamon, Oxford, pp. 123-177.

Richard Dupuis (2010). Application of oil debris monitoring for wind turbine gearbox prognostics and health management. Annual Conference of the Prognostics 
and Health Management Society, October 10-16, Portland, Oregon USA.

RWC Belgium World Data Center, Online sunspot data archive, SIDC, [Online]. Available: [Cited: $20^{\text {th }}$ Dec, 2012]: http://sidc.oma.be/index.php3.

S. Seneff (1988). A Joint Synchrony/mean-rate Model of Auditory Speech Processing. Journal of Phonetics, vol. 16, no.1, pp. 55-76.

T. Lin, B. G. Horne, P. Tino, and C. L. Giles (1997). A delay damage model selection algorithm for NARX neural networks. IEEE Transactions on Signal Processing, vol. 45, no. 11, pp. 2719-2730.

Uros Lotric and Andrej Dobnikar (2005). Predicting time series using neural networks with wavelet-based denoising layers. Neural Computing and Applications, vol. 14, pp. 11-17.

V. Indira, R. Vasanthakumari and V. Sugumaran (2010). Minimum sample size determination of vibration signals in machine learning approach to fault diagnosis using power analysis. Expert Systems with Applications, vol. 37, no.12, pp. 8650-8658.

Wang W. (2007). An adaptive predictor for dynamic system forecasting. Mechanical Systems and Signal Processing, vol. 21, no. 2, pp. 809-23.

Wang WQ (2004), Golnaraghi MF, Ismail F. Prognosis of machine health conditionusing neuro-fuzzy systems. Mech Syst Signal Process. Vol. 18, pp. 813-31.

Yaguo Lei, Ming J. Zuo, Zhengjia He, and Yanyang Zi (2010). A multidimensional hybrid intelligent method for gear fault diagnosis. Expert Systems with Applications, vol. 37, no. 2, pp. 1419-1430.

\section{BIOGRAPHIES}

Sajid Hussain is a PhD student in Electrical and Computer Engineering at University of Ontario Institute of Technology (UOIT) working under supervision of Dr. Hossam A. Gabbar. He did his diploma in Signal Processing from Aalborg University Denmark in 2003 and MSc in Telecommunication from Technical University Denmark in 2006. He worked as a Research Assistant in Computer Systems Engineering at Blekinge Institute of Technology, Sweden for two years. He is the author of more than 18 publications in the area of computer graphics, vibration analysis and risk-based maintenance. He also holds a patent in psychoacoustics for fault detection in electromechanical machines. He has 10 years of industrial experience in computer systems and machines condition monitoring.

Dr. Hossam A. Gabbar is Associate Professor in the Faculty of Energy Systems and Nuclear Science, and cross appointed in the Faculty of Engineering and Applied Science, University of Ontario Institute of Technology (UOIT). He obtained his Ph.D. degree (Safety Engineering) from Okayama University (Japan), while his undergrad degree (B.Sc.) is in the area of automatic control from Alexandria University, Egypt. He is specialized in safety and control engineering where he worked in process control and safety in research and industrial projects in Japan and Canada. Since 2004, he was tenured Associate Professor in the Division of Industrial Innovation Sciences at Okayama University, Japan. And from 2001, he joined Tokyo Institute of Technology and Japan Chemical Innovative Institute (JCII), where he participated in national projects related to advanced distributed control and safety design and operation synthesis for green energy and production systems. He developed new methods for automated control recipe synthesis and verification, safety design, and quantitative and qualitative fault simulation.

$\mathrm{He}$ is a Senior Member of IEEE, the founder of SMC Chapter - Hiroshima Section, the founder and chair of the technical committee on Intelligent Green Production Systems (IGPS), and Editor-in-chief of International Journal of Process Systems Engineering (IJPSE), president of RAMS Society, and editorial board of the technical committee on System of Systems and Soft Computing (IEEE SMCS). He is invited speaker in several Universities and international events, and PC / chair / co-chair of several international conferences. Dr. Gabbar is the author of more than 110 publications, including books, book chapters, patent, and papers in the area of safety and control engineering for green energy and production systems. His recent work is in the area of risk-based safety and control design for energy conservation and supply management, and smart grid modeling and planning with distributed generation. 